\title{
Use of Laser Measurements and Video Images to Investigate Pedestrian Movement along Non-Uniform Sidewalks
}

\author{
Mehmet Caputcu ${ }^{1}$, Burak Sengoz ${ }^{2}$, Mustafa Ozuysal' ${ }^{2}$, Serhan Tanyel $^{2}$, Sezin Kaplan ${ }^{3}$, Aykut Karabayir ${ }^{3}$ \\ ${ }^{1}$ Gediz University Department of Civil Engineering \\ Seyrek, Menemen, Izmir, Turkey \\ mehmet.caputcu@gediz.edu.tr \\ ${ }^{2}$ Dokuz Eylul University Department of Civil Engineering \\ Tinaztepe, Buca, Izmir, Turkey \\ burak.sengoz@deu.edu.tr, mustafa.ozuysal@deu.edu.tr, serhan.tanyel@deu.edu.tr \\ ${ }^{1,3}$ Dokuz Eylul University Graduate School of Natural and Applied Sciences-Department of Civil Engineering \\ Tinaztepe, Buca, Izmir, Turkey \\ sezukpln@gmail.com, aykutkarabayr@gmail.com
}

\begin{abstract}
While depending on various factors such as alignment of roads, location of and distance to central attractions, affordability of personal vehicle ownership, and social choices, pedestrian transportation mode often constitutes a significant portion of urban transportation demand. Accessibility to mass transportation systems, which are considered the most cost-effective mode of transportation, also necessitates walking. Therefore, performance of walking facilities is critical in achieving the goal of livable cities. On the other hand, some road sides with pedestrian traffic are often subject to a number of problems. Mostly being encountered in developing countries, these problems can be broadly classified as; absence of sidewalks along urban arterials or collectors, sidewalks with insufficient width for pedestrian traffic demand, and poor serviceability due to illegal fixed obstacles or unfixed encroachments. Furthermore, the comfort level and sense of safety are affected significantly. Investigation of these situations require research with use of advanced technology which is seemingly limited in those countries. Laser range scanners offer an affordable and yet robust data collection method for analyzing complex traffic scenes with undesired interactions. This paper presents a method as an alternative to conventional data collection methods for traffic analysis, and suggests that it can be adapted to investigate micro-interactions and conflicts around locations of aforementioned sidewalk problems.
\end{abstract}

Keywords: sidewalk problems, data collection with laser scanners, walking speeds, pedestrian attributes, regression model

\section{Introduction}

Scientific research studies on different aspects of pedestrian movements and mobility are numerous. Characteristics of pedestrian flow, methods of pedestrian data collection, pedestrian behavior and factors of their choices, performance of sidewalks as transportation infrastructure, modeling of pedestrian movements with different approaches, criteria for evaluating sidewalk environment, physical problems on sidewalks, undesired interactions and conflicts between pedestrian traffic and vehicular traffic, pedestrian level of service, simulation of pedestrian movements, and use of new technologies in pedestrian research are some main categories of studies conducted by researchers from various disciplines.

Vuchic [1] pointed out that personal cars are becoming excessively used in modern city life and this habit turns into addiction. He emphasized that the level of comfort and safety a pedestrian or bicyclist feels is a significant indicator of livability in an urban area. Oral [2] reported that pedestrian transportation (walking) constitutes 30 35\% of total daily transportation demand in the city of Izmir, Turkey. Therefore, the performance and appropriate design of walking facilities are critically important.

The "walk score" walkability index method, which has also given its name to a firm, is an evaluation approach based on the distance of a point (such as the address of a house) to the nearest public attractions for business, shopping, entertainment, education, health, etc. However, this method does not take into account the physical conditions along sidewalks to those attraction points [3-4].

Protection and sustainability of human health is another major issue that tightly relates walking. Increase in car ownership and free commuting transport provided by employers makes walking a key activity for a healthier and longer life. 
The concept of "active living", which can be described as "a way of life that integrates physical activity into everyday routines, such as walking for transportation" [3], arises against the global problem of decline in mode share of walking [5].

Mostly observed in cities of developing countries, on the other hand, walking discomfort and traffic safety risks due to deficiencies in sidewalk design and applications stand out as a barrier for pedestrians despite an adopted tendency to walk in these communities. Inappropriate physical conditions or absence of sidewalks along some major urban roads force pedestrians to experience conflicts with the parallel-moving vehicular traffic. In the context of pedestrian-vehicle conflicts, many studies have focused on situations where pedestrians cross vehicular road, such as crossings at intersections, midblock crossings, and other crossing movements outside of designated (legal) crossings [6-18]. Very few studies have investigated the interactions between adjacent pedestrian traffic and vehicular traffic which both move parallel to each other along a road, lacking a quantitative approach with high precision.

Current study presents the first stage of a novel approach to determine losses and delays experienced by pedestrians due to sidewalk problems which could primarily be classified as (1) absence of a sidewalk on the road used by pedestrians, (2) insufficient width of a sidewalk causing some pedestrians to use pavement when sidewalk is occupied by and has to be shared with another (group of) pedestrian(s), and (3) fixed or unfixed obstacles which narrow the effective width of sidewalk (Fig. 1). The broader study aims to determine delays and other losses (comfort, level of service, safety, etc.) of pedestrians exposed to these problems, by using sensitive laser measurements to compute various spatiotemporal variables. This paper focuses on pedestrian movements on a sidewalk which is considered as compliant with standards (a control site). The data from this site will be utilized in a future analysis as a basis for a comparison with the same pedestrian characteristics and environment conditions observed in other sites with aforementioned sidewalk problems (experimental sites).

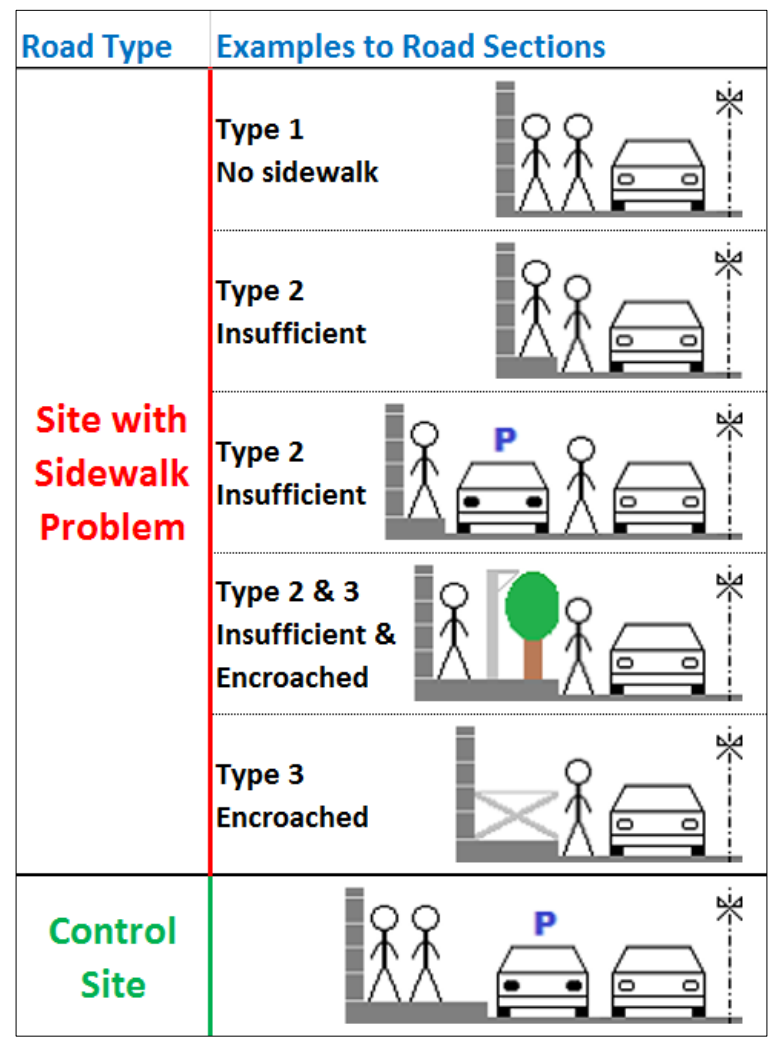

Fig. 1: Illustration of sites with and without sidewalk problems.

\section{Methodology}

The site evaluated in this paper is the sidewalk on Erdem Avenue across the Dokuzcesmeler Campus of Dokuz Eylul University in Izmir, as presented in Fig. 2. It was planned to serve as the control site for the experimental sites with sidewalk problems that will be investigated in a future study. Data collection at this site was performed one weekday every month 
within one year. Three SICK laser range measurement devices (LMS511) scanning a horizontal plane were utilized to record a plan of pedestrian and vehicle movements, as well as other stationary or moving objects every $40 \mathrm{~ms}(25 \mathrm{~Hz})$.

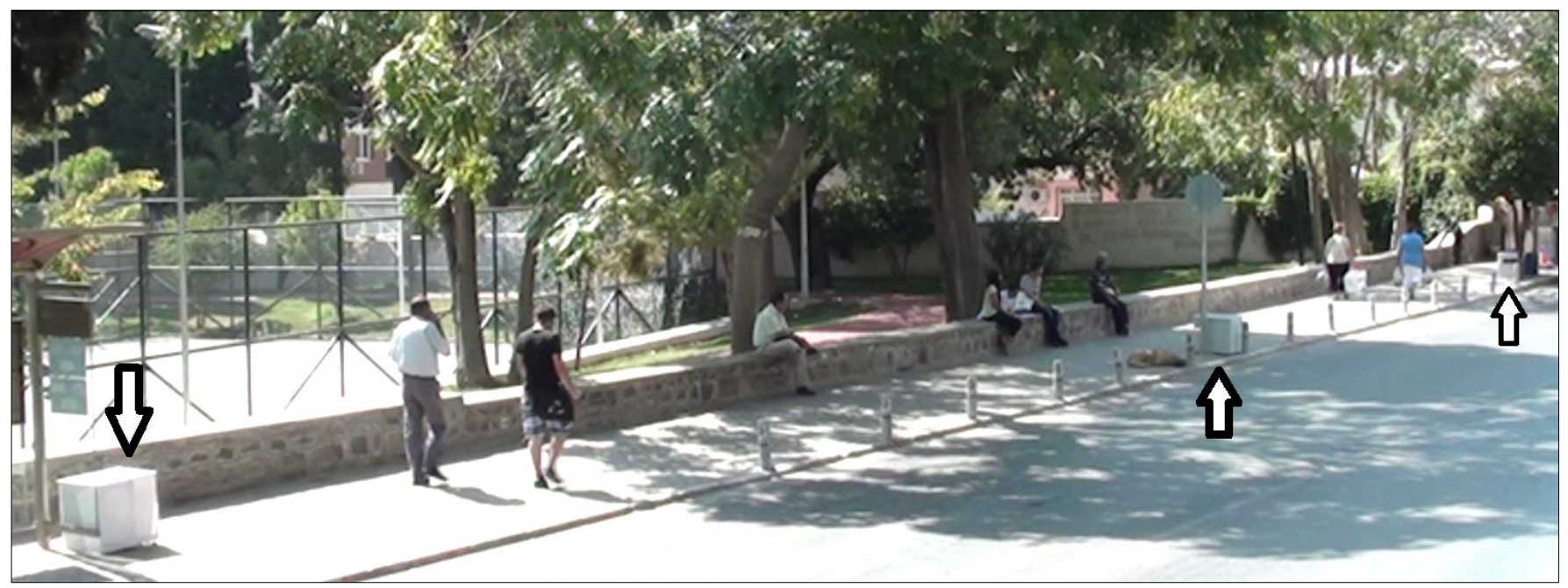

Fig. 2: The site of investigation and location of laser scanners (devices marked with an arrow).

The devices were located such that obstructions were minimized (Fig. 3). Two-dimensional point cloud scanned by these devices were saved as polar coordinates on the site and transformed into Cartesian coordinates with further processing in the office. Laser scanning was accompanied by video recordings covering the extents of the site being investigated. Various pedestrian attributes such as (approximate) "age group", "weight", "load status (e.g. whether carrying a bag etc.)", "whether paid attention to measurement equipment" were extracted from these video records, then were associated with spatiotemporal information obtained through the following procedure.

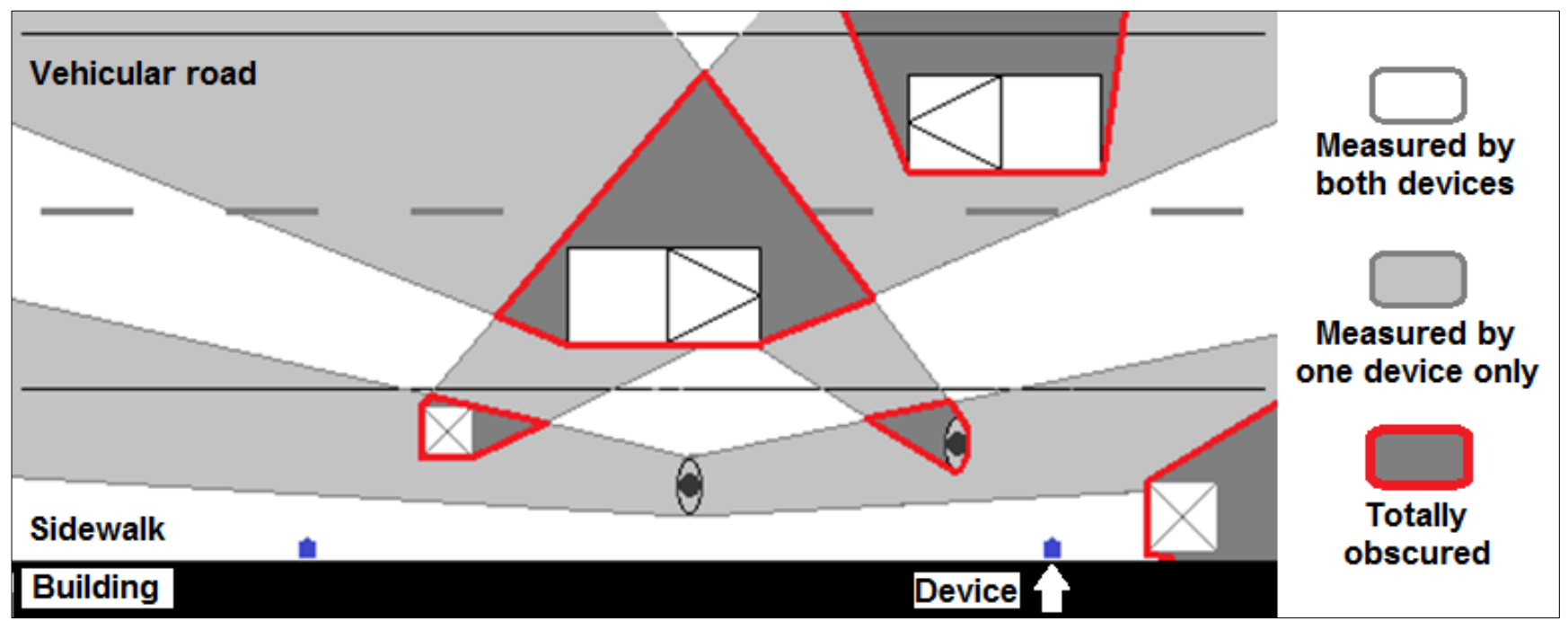

Fig. 3: Illustration of areas covered in a case of multiple laser measurement devices.

Following the conversion from polar to Cartesian coordinates, the data were in the form of a three-dimensional matrix, as illustrated in Fig. 4. Devices were set to scan, in other words send a laser beam, every $0.25^{\circ}$ within their respective scanning planes, which yielded to 761 measurements in one time frame since they scan between $-5^{\circ}$ and $+185^{\circ}$ (Fig. 5). 


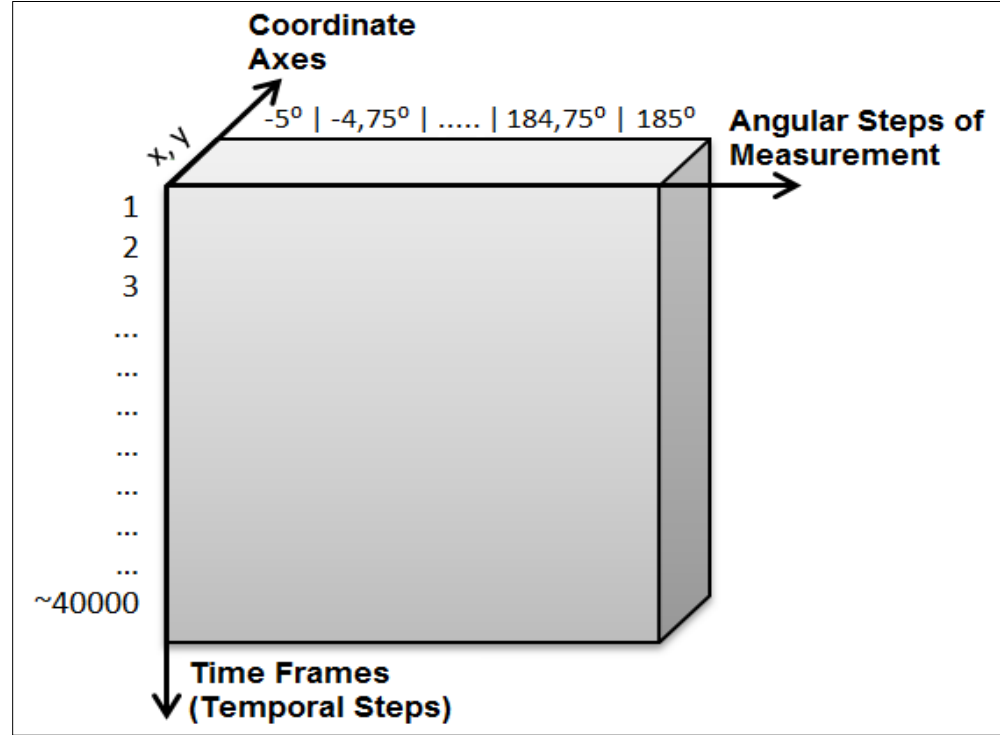

Fig. 4: Form of the matrix used in tracing pedestrians and vehicles.

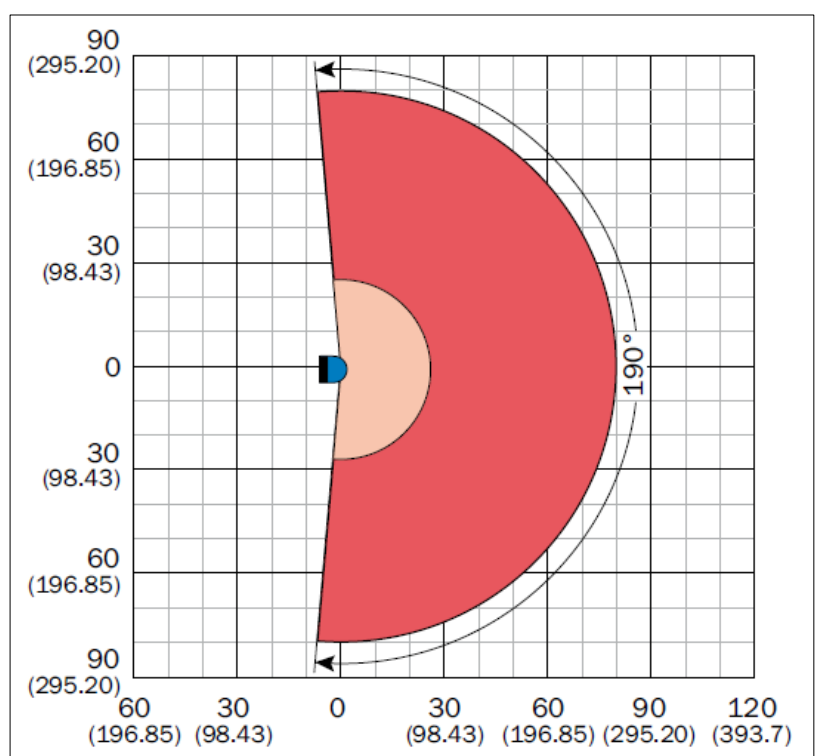

Fig. 5: Scanning range in $\mathrm{m}$ (feet) (Source: Specifications of the product).

The data were undergone a background subtraction process to classify points as "moving" and "stationary". This was a necessary step to lessen processing load due to high number of points in every frame and to avoid potential noises while tracing movement of users. The method of background subtraction was based on histograms giving the occurrence of unique distance measurements at each angular step of laser scanners. Histogram of every angular step of each device were examined through computation separately. Fig. 6 shows a traffic scene with pedestrians walking on a sidewalk and vehicles using the adjacent road. Numbers on the picture correspond to those in Fig. 7 which shows the histogram of distance measurements obtained in about thirty minutes from one of the laser beams crossing the road.

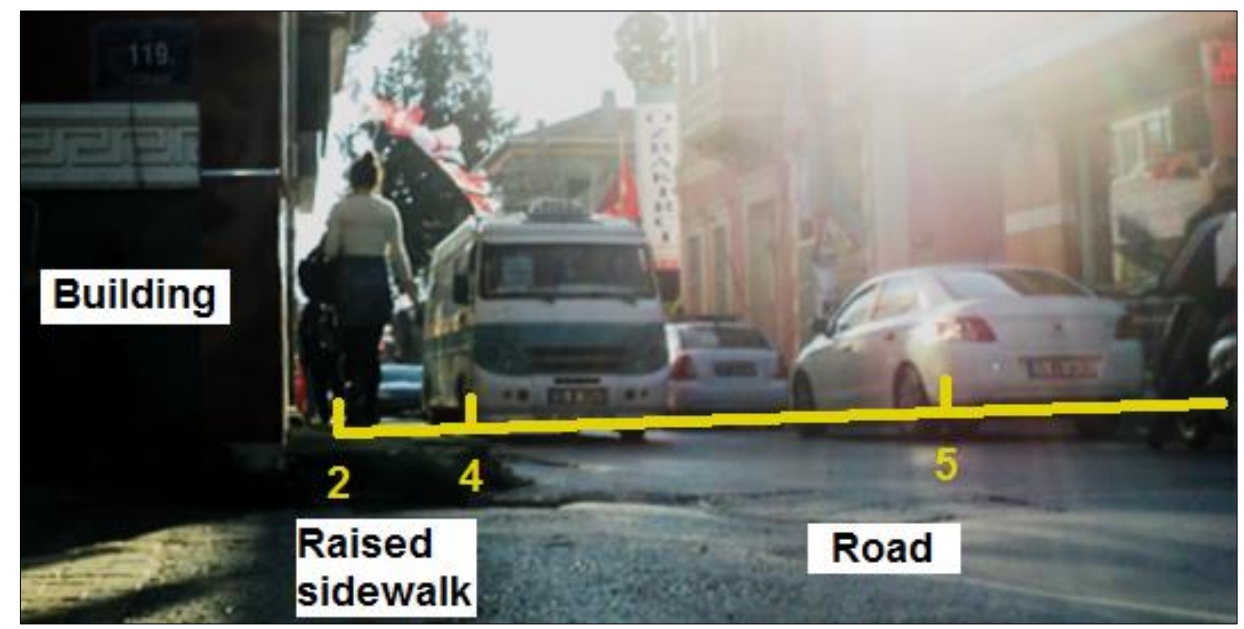

Fig. 6: Moving objects (sidewalk and road users) along the cross-section of a road (another site). 


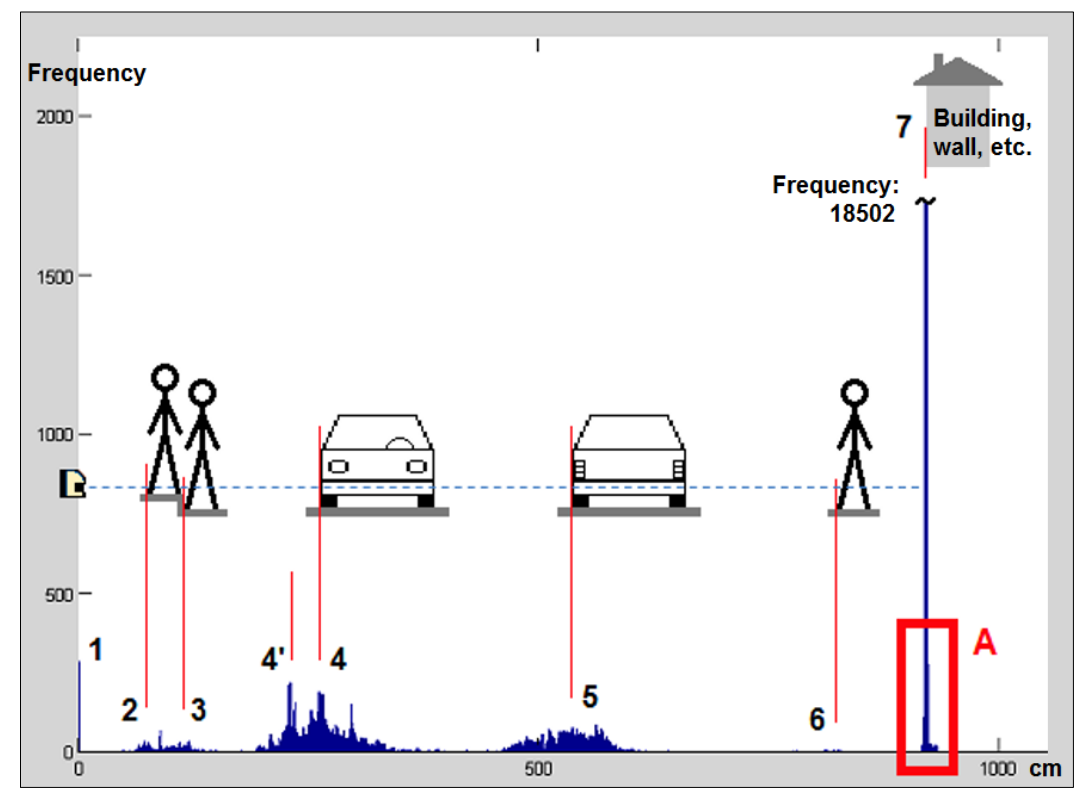

(a)

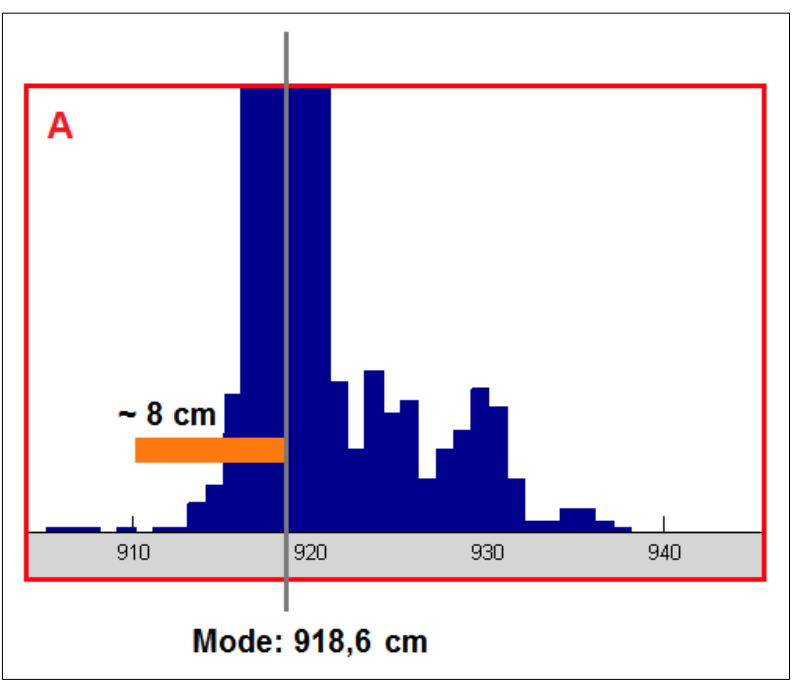

(b)

Fig. 7: (a) Histogram of measured distances and objects yielding those measurements.

(b) Detail of region A and mode value of distance measurements.

Considering the devices' margins of error at varying distances (Fig. 8) and by checking the spread of measurements around the mode value at different angular steps (Fig. 7.b), a threshold was assigned to every angle. Using the threshold value, points were classified as moving or stationary and exported into separate matrices. This procedure was repeated for each of the three measurement device used at the site. The outputs were two different data tables one of which included laser points of pedestrians, vehicles, and any other moving object in the traffic scene while the other included that of structures, poles, bus stops, walls, trees, etc.

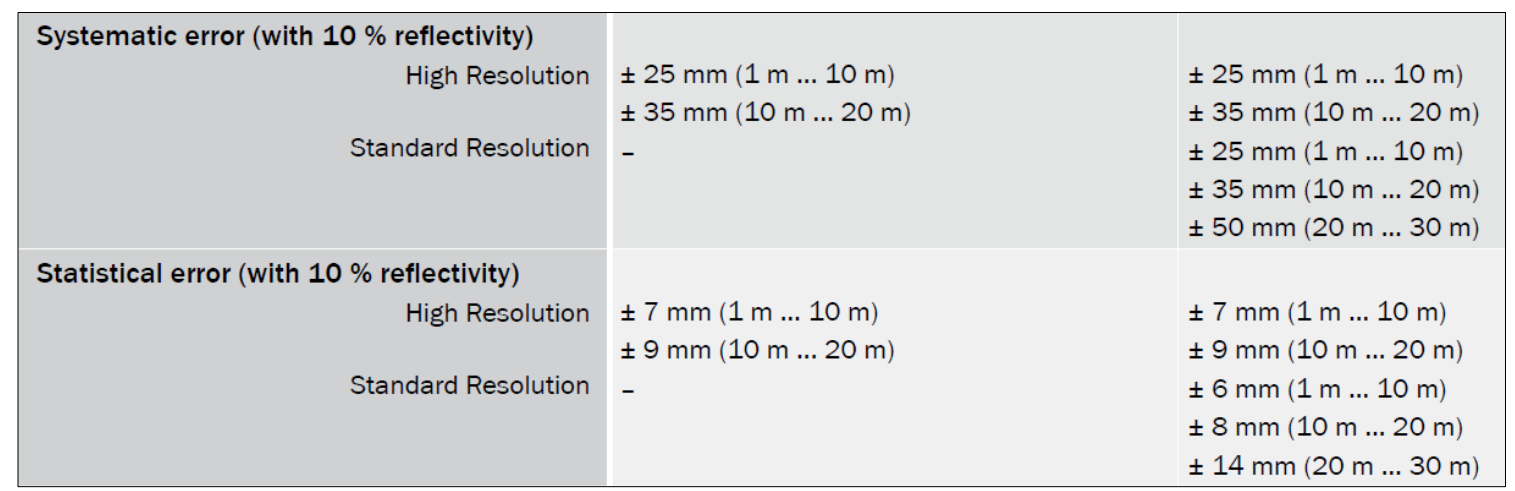

Fig. 8: Measurement error margins of SICK LMS511 (Source: Specifications of the product).

Since three laser devices were used, the above process resulted in three different sets of moving and stationary object matrices. The next step was combining three separate matrices into one single matrix to be used as input for tracing users in traffic. Relative positions and coordinates of measurement devices were found by overlaying their respective laser points in AutoCAD. The one in the middle out of three devices was selected as the base device, then coordinates from other devices were transformed and appended onto base device's data table by creating common temporal bins as the timestamps. One critical issue at this stage was efficient synchronization of timestamps for correct association of records. Computers attached to each device during data collection had already been synchronized by means of an online tool with a sensitivity level of 10 $\mathrm{ms}$ or better. However, later in the laboratory it was found out that in some cases there were discrepancies across timestamps of devices, between one another. A shift of even a few milliseconds while integrating data could affect the performance of 
tracking negatively. In order to overcome this problem, data from different devices were tentatively combined using color codes and then monitored using Matlab's visualization tool. By manual intervention to timestamps, all three matrices of moving points were synchronized to a satisfactory level. Fig. 9 shows the laser image of a moving box (pulled with rope) in an experiment conducted in the laboratory, where (a) shows wrong association and (b) is the result after calibration.

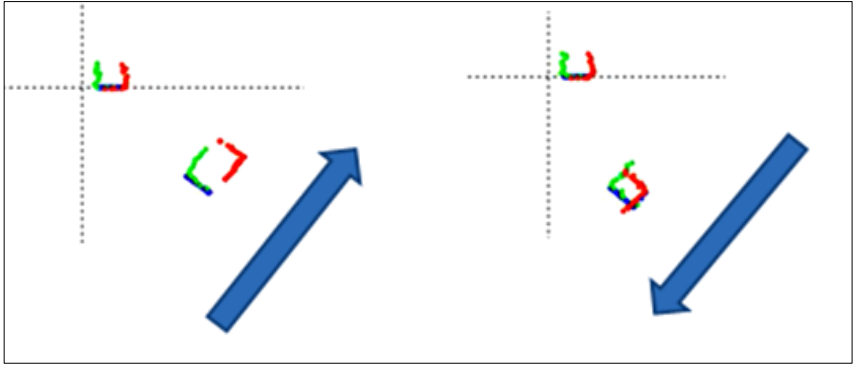

(a)

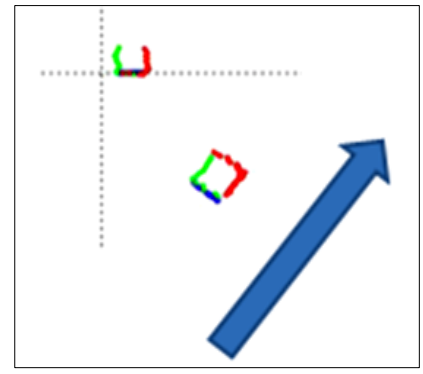

(b)

Fig. 9: Calibration of timestamps during integration of multiple data sources.

After temporal association between different devices were checked and manual calibrations were made as necessary, moving points from each device and stationary objects were combined into one matrix each, respectively. Then the matrix of moving objects and that of the fixed objects were visualized for evaluation as seen in Fig. 10.

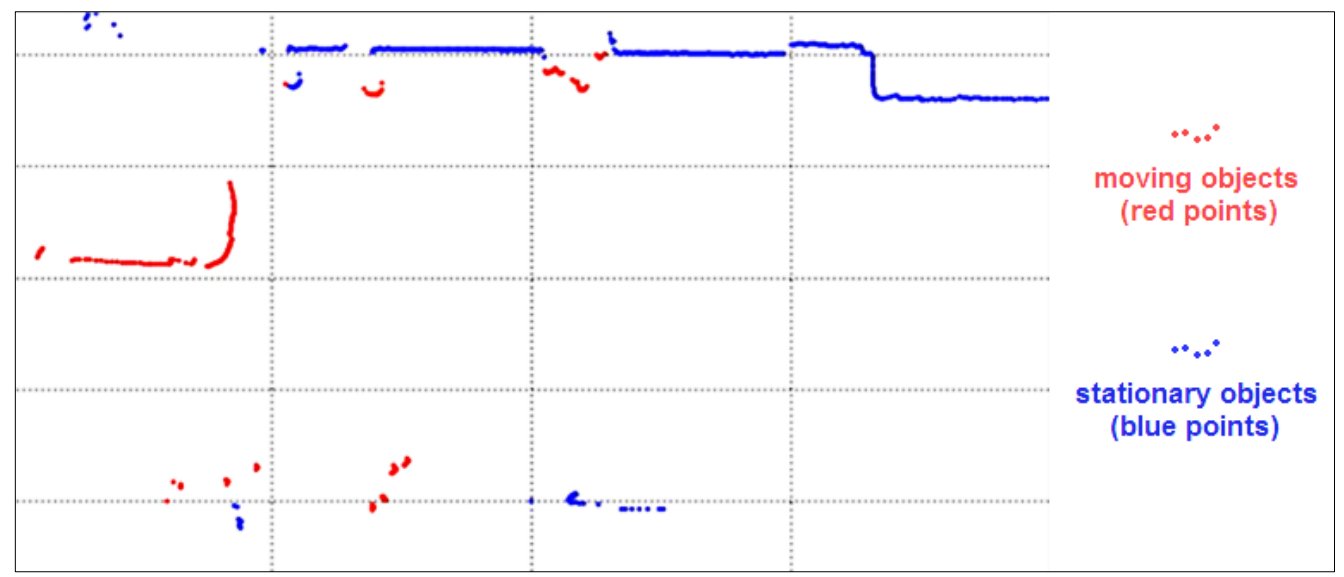

Fig. 10: A traffic scene with a vehicle and multiple pedestrians after background subtraction.

In the final step of data processing stage, one section at both ends of the scanned region on the sidewalk were determined, then timestamp and coordinates of pedestrians passing both sections with a steady walk were recorded while those with undesired irregular movements and paths were excluded. The final output included location and time of pedestrians which would allow computation of their average walking speeds within the desired interval, as well as other attributes mentioned earlier.

\section{Analysis and Results}

A regression model was estimated using the data obtained from the method described above. Data from 8 months out of 11 were used in this analysis. These months included 2 months representing each of the four seasons. Attributes from both laser scans and video images were evaluated together. A total number of 1027 pedestrians were truncated down to 997 after eliminating outliers, errors, missing variables, and other odd cases. Available variables included month of year (season), day of week, hour of day, temperature, humidity, direction of pedestrian, gender, age group, weight, type of clothing, load status, load type, walking pace, group walking, interaction with measurement equipment, and average speed within the desired interval.

Table 1 shows descriptive statistics for selected variables which were found to be significant in the model. 
Table 1: Descriptive statistics of selected variables.

\begin{tabular}{|l|c|l|l|c|c|}
\hline Variable & $\begin{array}{c}\text { Number of } \\
\text { Pedestrians }\end{array}$ & $\begin{array}{c}\text { Average Speed } \\
(\mathbf{m} / \mathbf{s})\end{array}$ & Variable & $\begin{array}{l}\text { Number of } \\
\text { Pedestrians }\end{array}$ & $\begin{array}{c}\text { Average Speed } \\
\text { (m/s) }\end{array}$ \\
\hline Gender & & & Load & \\
\hline Male & 573 & 1.339 & Heavy & 25 & 1.197 \\
\hline Female & 424 & 1.273 & Moderate & 165 & 1.315 \\
\hline Age Group & & & Light & 478 & 1.303 \\
\hline-15 & 13 & 1.117 & None & 329 & 1.330 \\
\hline $15-25$ & 747 & 1.338 & Weight & & \\
\hline $25-40$ & 117 & 1.300 & Overweight & 106 & 1.235 \\
\hline $40+$ & 120 & 1.173 & Normal & 798 & 1.314 \\
\hline Temperature & & & Slim & 93 & 1.370 \\
\hline$<20^{\circ} \mathrm{C}$ & 591 & 1.314 & Group Walking & & \\
\hline $20-25^{\circ} \mathrm{C}$ & 164 & 1.313 & 1 person & 545 & 1.363 \\
\hline $25-30^{\circ} \mathrm{C}$ & 108 & 1.304 & 2 persons & 323 & 1.268 \\
\hline $30+{ }^{\circ} \mathrm{C}$ & 134 & 1.301 & 3 persons & 100 & 1.235 \\
\hline Grand Total & 997 & 1.311 & $4+$ person & 29 & 1.084 \\
\hline & & & Grand Total & 997 & 1.311 \\
\hline
\end{tabular}

Speed was selected as the dependent variable of the model. After six iterations, the model was estimated as presented in Table 2. The output of the analysis shows that people tend to walk faster when weather is colder. Results also approve the expected case that males walk faster than females while speed increases when age is below 25 or the person is thin. Walking as groups affect speed negatively, with speed decreasing as number in the group raises.

Table 2: Results of the regression estimation model.

\begin{tabular}{|c|c|c|c|c|c|}
\hline Dependent Variable: Walking Speed & Coeffecients & Std. Error & $p$-value & & \\
\hline (Constant) & 1.162 & 0.021 & 0.0000 & \multirow{3}{*}{$\mathrm{R}$} & \multirow{3}{*}{0.528} \\
\hline Temperature $<20^{\circ} \mathrm{C}$ & 0.019 & 0.011 & 0.0934 & & \\
\hline Gender: Male & 0.064 & 0.012 & 0.0000 & & \\
\hline Age group: $15-25$ & 0.222 & 0.018 & 0.0000 & \multirow{3}{*}{ R Square } & \multirow{3}{*}{0.279} \\
\hline Age group: $25-40$ & 0.149 & 0.022 & 0.0000 & & \\
\hline Weight: Slim & 0.049 & 0.019 & 0.0108 & & \\
\hline Weight: Overweight & -0.031 & 0.018 & 0.0946 & \multirow{3}{*}{$\begin{array}{l}\text { Adjusted R } \\
\text { Square }\end{array}$} & \multirow{3}{*}{0.271} \\
\hline Load: Moderate & -0.038 & 0.014 & 0.0059 & & \\
\hline Load: Heavy & -0.035 & 0.018 & 0.0484 & & \\
\hline Group walking: 2 persons & -0.107 & 0.012 & 0.0000 & \multirow{3}{*}{$\begin{array}{l}\text { Std. Error of } \\
\text { the Estimate }\end{array}$} & \multirow{3}{*}{0.169} \\
\hline Group walking: 3 persons & -0.160 & 0.019 & 0.0000 & & \\
\hline Group walking: 4 persons & -0.332 & 0.033 & 0.0000 & & \\
\hline
\end{tabular}

An unexpected result was about the effect of load status on walking speed. Based on the model it can be concluded that moderate-level load yields a higher drop in the estimated speed than heavy load does. Descriptive statistics showing relation of frequency and speed to load type (handbag, shopping sack, book/notebook in hand, backpack, etc.) were checked to investigate the underlying reason. It was found that backpack was categorized under heavy load and pedestrians with a backpack who seemed to be a high school or university student walked faster. This finding might also point to a relation between tendency for active living [3] and using sports utility clothing.

One potential problem with this study was the risk of pedestrian distraction because of the 'white boxes', the measurement equipment along the sidewalk, during data collection sessions. According to video records, only $11 \%$ of 997 
pedestrians in the analysis seemed to have noticed the existence of these devices. Unexpectedly, their speeds did not demonstrate any drop, as shown in

Table 3. Thus, the effect of existence of devices were neglected.

Table 3: Relation between speed and distraction by laser device.

\begin{tabular}{|l|c|c|}
\hline Interaction With Measurement Equipment & Number of Pedestrians & Average Speed (m/s) \\
\hline Turned head and looked & 114 & 1.333 \\
\hline Doesn't seem to have noticed & 883 & 1.307 \\
\hline Grand Total & 997 & 1.310 \\
\hline
\end{tabular}

The average speed values obtained in this study complies with past findings available in the literature. For example, Hughes [19] and Toshiyuki [20] provided a three-dimensional representation showing the cross-relations between pedestrians' walking speed, density of parallel-moving pedestrian flow, and density of pedestrian flow with crossing movement. The experiment described in this paper focused on a sidewalk where no crossing flow is assumed and density of parallel flow in both directions does not exceed 1 person $/ \mathrm{m} 2$. Thus, according to the chart in

Fig. 11, an average walking speed of between 1.28-1.4 m/s (marked on the figure) would be expected to be observed in this study, and it was achieved.

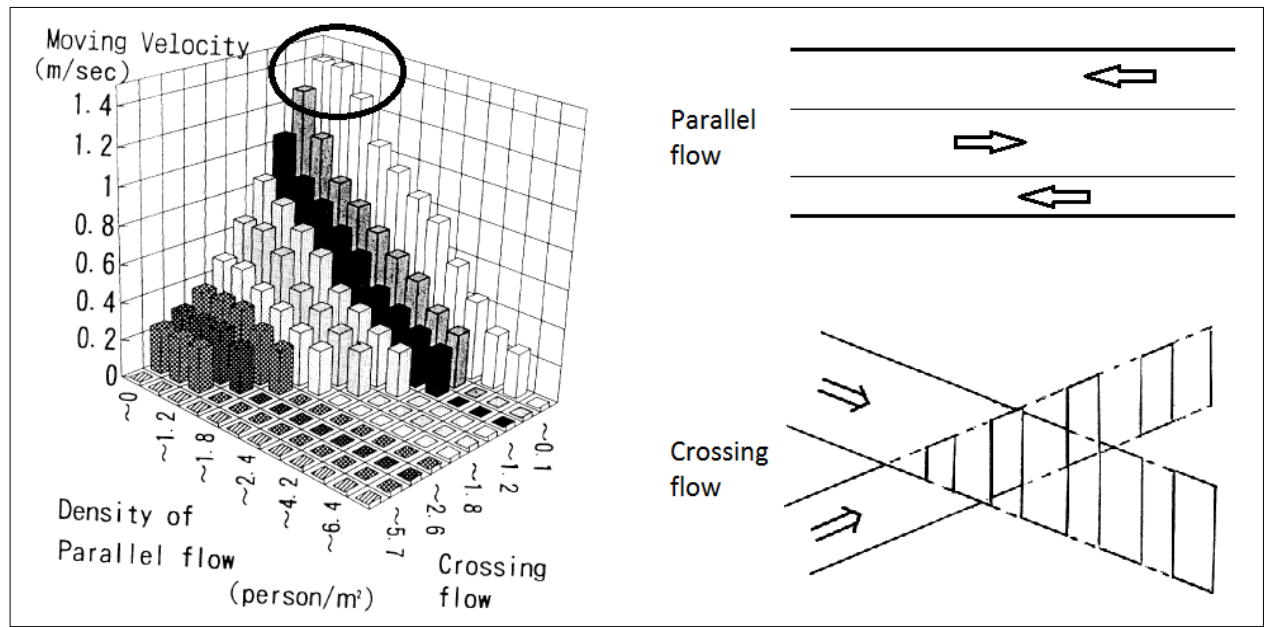

Fig. 11: Three-dimensional plot of pedestrian speed as a function of flow density and crossing flow density.

(Adapted from Hughes [19], Toshiyuki [20], and Ando et al. [21].)

\section{Conclusions and Discussion}

With respect to precision, use of laser measurements in traffic studies have superiority over conventional data collection methods such as use of stopwatches on a constant point or between two constant sections. Furthermore, utilization of laser points offer the flexibility to extract timestamps and locations (coordinates) at any desired point within the measured range, allowing the computation of variables such as speed, direction and density.

As indicated by Shao et al. [22], laser scanners offer some advantages including; direct measurement of distances and recording positions in real dimension, high accuracy and stability due to being free of illumination, color, or texture effects, speed and ease of data collection, possibility of covering a large area by integrating multiple scanners, ability to evaluate crowded scenes due to low computation cost, and elimination of privacy issues. The only caveat with the method is the care required and time consumed for successful classification of moving versus stationary objects and integration of data from different devices.

This paper presented a study of pedestrian speeds precisely computed from range measurements in the unit of centimeters and timestamps in milliseconds. In the next stage of this ongoing research study, authors plan to apply this method to traffic scenarios at other sites demonstrating sidewalk problems defined in the Introduction section. The method 
promises to provide information about micro-reactions and -behaviors of pedestrians against various obstacles along a corridor, with implications on pedestrian delay, level of service, traffic conflict technique (TCT), safety, and effects on vehicular traffic. A follow up study may yield to inferences on possible effects of sidewalk problems on transportation mode choice of people living in urban areas, such as discouraging from walking.

\section{Acknowledgements}

This study was conducted as part of the research project "Analysis of Pedestrian-Vehicle Conflicts Caused by Design and Implementation of Pedestrian Facilities" funded by TUBITAK 1001 Grant Program with project number $113 \mathrm{M} 222$. Authors would like to thank TUBITAK for making it possible, to Dr. Xiaowei Shao for his guidance in the data collection and processing stage, to Dr. Haidar Sharif, Feyza Galip, and Hilal Inan for their support, and to undergraduate and graduate students from Dokuz Eylul University and Gediz University for their assistance during data collection.

\section{References}

[1] V. R. Vuchic, Transportation for Livable Cities. Center for Urban Policy Research, 2005.

[2] M. Y. Oral, "Izmir Transportation Master Plan 2009," Ege Mimarlık, pp. 42-48, 2012.

[3] E. Cubukcu, B. Hepguzel, Z. Onder, and B. Tumer, "Active Living for Sustainable Future: A model to measure "walk scores" via Geographic Information Systems," Procedia - Social and Behavioral Sciences, vol. 168, pp. 229 - 237, 2015.

[4] Walk Score [Online]. Available: https://en.Wikipedia.org/wiki/Walk_Score\#Walk_Score.

[5] E. Cubukcu, "Walking for Sustainable Living," Procedia - Social and Behavioral Sciences, vol. 85, pp. 33-42, 2013.

[6] M. J. Cynecki, "Development of Conflicts Analysis Technique for Pedestrian Crossings," Transportation Research Record, vol. 743, pp. 12-20, 1980.

[7] B. L. Bowman and R. L. Vecellio, "Pedestrian Walking Speeds and Conflicts at Urban Median Locations," Transportation Research Record, vol. 1438, pp. 67-73, 1994.

[8] H. N. AbdulSattar, M. S. Tarawneh, P. T. McCoy, and S. D. Kachman, "Effect on Vehicle-Pedestrian Conflicts of "Turning Traffic Must Yield to Pedestrians" Sign,” Transportation Research Record, vol. 1553, pp. 38-45, 1996.

[9] D. Lord, "Analysis of Pedestrian Conflicts with Left-Turning Traffic," Transportation Research Record, vol. 1538, pp. 61-67, 1996.

[10] J. S. Milazzo, N. M. Rouphail, J. E. Hummer, and D. P. Allen, "Effect of Pedestrians on Capacity of Signalized Intersections," Transportation Research Record, vol. 1646, pp. 37-46, 1998.

[11] R. V. Houten, J. E. L. Malenfant, and D. McCusker, "Advance Yield Markings Reduce Motor Vehicle/Pedestrian Conflicts at Multilane Crosswalks with an Uncontrolled Approach," Transportation Research Record, vol. 1773, pp. 69-74, 2001.

[12] S. Malkhamah, M. Tight, and F. Montgomery, "The Development of an Automatic Method of Safety Monitoring at Pelican Crossings, Accident Analysis \& Prevention," Elsevier, vol. 37, no. 5, pp. 938-946, 2005.

[13] D. Akin and V. P. Sisiopiku, "Modeling Interactions Between Pedestrians and Turning Vehicles at Signalized Crosswalks Operating Under Combined Pedestrian-Vehicle Interval," in TRB 86th Annual Meeting, Transportation Research Board, Washington, D.C., 2007, pp. 19.

[14] W. Junhua and F. Shouen, "Pedestrian-Vehicle Conflict Observation and Characteristics of Road Section," Natural Science Journal of Tongji University, vol. 4, pp. 503-507, 2008.

[15] Y. Chen, H. Meng, and Z. Wang, "Safety Improvement Practice for Vulnerable Road Users in Beijing Junctions," in TRB 86th Annual Meeting, Transportation Research Board, Washington, D.C., 2009, pp. 10.

[16] K. Ismail, T. Sayed, N. Saunier, and C. Lim, "Automated Analysis of Pedestrian-Vehicle Conflicts Using Video Data," Transportation Research Record, vol. 2140, pp. 44-54, 2009.

[17] K. Ismail, T. Sayed, and N. Saunier, "Automated Analysis of Pedestrian-Vehicle Conflicts: A Context for Before-andAfter Studies," Transportation Research Record, vol. 2198, pp. 52-64, 2010.

[18] W. Junhua, "Pedestrian's Critical Cross Gap and Its Application in Conflict Simulation," in International Conference on Intelligent Computation Technology and Automation (ICICTA), IEEE, 2010, vol. 2, pp. 889-892.

[19] R. L. Hughes, A Continuum Theory For The Flow Of Pedestrians. 2002.

[20] A. Toshiyuki, "Prediction system of passenger flow," in Engineering for Crowd Safety, R. A. Smith and J. F. Dickie, Ed., Amsterdam: Elsevier, 1993, pp. 249-258. 
[21] T. Ando, H. Ota, and T. Oki, "Forecasting the flow of people (in Japanese)," Railway Res. Rev., vol. 45, pp. 8-14, 1988.

[22] X. Shao, H. Zhao, K. Nakamura, K. Katabira, R. Shibasaki, and Y. Nakagawa, "Detection and Tracking of Multiple Pedestrians by Using Laser Range Scanners," in International Conference on Intelligent Robots and Systems (IROS 2007), IEEE/RSJ, 2007, p. 2174-2179. 